\title{
Carriage of two carbapenem-resistance genes in Pseudomonas aeruginosa isolated from hospital-acquired infections in children from Costa Rica: the importance of local epidemiology
}

\author{
Cristian Pérez-Corrales ${ }^{*} \mathbb{0}$, Valeria Peralta-Barquero and Christopher Mairena-Acuña
}

\begin{abstract}
Background: The assessment of Hospital-acquired infections due to multidrug-resistant bacteria involves the use of a variety of commercial and laboratory-developed tests to detect antimicrobial resistance genes in bacterial pathogens; however, few are evaluated for use in low- and middle-income countries.

Methods: We used whole-genome sequencing, rapid commercial molecular tests, laboratory-developed tests and routine culture testing.

Results: We identified the carriage of the metallo- $\beta$-lactamase bla $a_{\mathrm{VIM}-2}$ and $b / a_{\mathrm{IMP}-18}$ alleles in Carbapenem-Resistant Pseudomonas aeruginosa infections among children in Costa Rica.

Conclusions: The bla $a_{1 \mathrm{MP}-18}$ allele is not present in the most frequently used commercial tests; thus, it is possible that the circulation of this resistance gene may be underdiagnosed in Costa Rica.
\end{abstract}

Keywords: Carbapenem-Resistant Pseudomonas aeruginosa, Hospital-acquired infections

\section{Background}

The global increase of infections due to multidrug-resistant bacteria remains a public health and sustainable development problem [1]. The global antimicrobial resistance surveillance system (GLASS) encourages healthcare authorities to increase laboratory capacity. To improve surveillance and diagnostic stewardship, GLASS also recommends the implementation of rapid, accurate diagnostic testing for antibiotic resistance [2]. The methods for the detection of resistance mechanisms are diverse,

${ }^{*}$ Correspondence: cperezc@ccss.sa.cr

División de Microbiología, Laboratorio Clínico, Hospital Nacional de Niños "Dr. Carlos Sáenz Herrera", Centro de Ciencias Médicas de La Caja

Costarricense del Seguro Social, PO Box: 1654-1000, San José, Costa Rica ranging from phenotypic to more complex genotypic tests, and whole-genome sequencing (WGS). The continuous development of diagnostic tests in and for high-income countries makes them available for other countries at a good price. However, information on antimicrobial resistance in low- and middle-income countries is scarce, as is the performance of these kits in different epidemiological contexts. Carbapenem-Resistant Pseudomonas aeruginosa (CRPA) is frequently isolated from healthcare-associated infections (HAI). The mechanisms of CRPA resistance can be driven by porin loss, efflux pump activity, or by horizontal gene transfer encoded in plasmids [3]. In Costa Rica, the National Children's Hospital is a tertiary referral hospital within the socialized medical care system. HAI assessment has

c) The Author(s) 2021. This article is licensed under a Creative Commons Attribution 4.0 International License, which permits use, sharing, adaptation, distribution and reproduction in any medium or format, as long as you give appropriate credit to the original author(s) and the source, provide a link to the Creative Commons licence, and indicate if changes were made. The images or other third party material in this article are included in the article's Creative Commons licence, unless indicated otherwise in a credit line to the material. If material is not included in the article's Creative Commons licence and your intended use is not permitted by statutory regulation or exceeds the permitted use, you will need to obtain permission directly from the copyright holder. To view a copy of this licence, visit http://creativecommons.org/licenses/by/4.0/. The Creative Commons Public Domain Dedication waiver (http://creativecommons.org/publicdomain/zero/1.0/) applies to the data made available in this article, unless otherwise stated in a credit line to the data. 
recently improved in this hospital with the implementation of a diagnostic stewardship program, which was established according to its needs and resources. To better understand CRPA circulation in Costa Rica and compare detection methods, we studied $P$. aeruginosa isolated from pediatric patients with HAI between late 2018 and 2020 using conventional phenotypic methods, rapid molecular test, and WGS.

\section{Methods}

Samples from patients with suspected HAI were collected and transported to the laboratory following the general specimen collection and transport techniques [4]. A total of $32 P$. aeruginosa isolates were analyzed using all of the following methods: conventional phenotypic methods, molecular tests, and whole-genome sequencing. Identification and susceptibility to antimicrobials were performed by automated Vitek-2 Systems (BioMérieux, Marcy-l'Étoile, France) using CLSI breakpoints for Carbapenems (MIC $\geq 8 \mu \mathrm{g} / \mathrm{mL}$ ) [5]. Phenotypic studies included modified carbapenem inhibition test (mCIM) according to CLSI [5] and metallo-ß-lactamase (MBL) E-test (BioMérieux, Marcy-l'Étoile, France). Molecular analyses for detection of genes conferring resistance included Xpert Carba Test (Cepheid, Sunnyvale CA, USA) for detection of genes encoding carbapenemases KPC, VIM, IMP, NDM, OXA-48; AMR Flow-Chip hybridization (Master Diagnóstica, Granada, Spain) for detection of genes encoding extended-spectrum betalactamases SHV, CTX-M; class A carbapenemases GES, SME, KPC, IMI; class B carbapenemases SIM, GIM, SPM, NDM, VIM, IMP and class D carbapenemases OXA23like, 24-like, 48-like, 51-like and 58-like; and detection of genes encoding carbapenemases IMP and VIM by Polimerase Chain Reaction (PCR) Laboratory-Developed Tests (LDT) using primers described elsewhere [6]. When required, DNA was extracted using MagNA Pure (Roche Diagnostics, Basel, Switzerland), quantified by Quantus (Promega, Madison WI, USA), and verified by Qiaxcell (QIAgen, Germantown MD, USA). The whole genome was prepared using the Nextera Flex library preparation standard protocol and paired-end fragment (151 bp) sequencing (MiSeq, Illumina Inc., San Diego CA, USA). A general pipeline for read quality (FastQC) [7], trimming (Trimmomatic) [8], genome assembly (Shovill) [9], genome annotation (Prokka) [10] and resistance-gene finder (ABRicate) [11] was performed using Galaxy Community Hub [12].

\section{Results}

A total of 8 out of the 32 bacterial isolates identified as $P$. aeruginosa exhibited resistance to imipenem and meropenem by automated means. In all 8 strains, mCIM was positive, indicating carbapenemase activity. Furthermore, MBL E-test methods confirmed the carbapenem resistance due to the presence of metallo- $\beta$-lactamase in all strains. The molecular methods classified all 8 strains in agreement with the observed phenotypic assays. However, GenXpert Carba RT-PCR, as well as the reversehybridization assay showed positive results for $b l a_{\mathrm{VIM}}$ detection only. Conventional PCR using LDT detected both $b l a_{\mathrm{VIM}}$ and $b l a_{\mathrm{IMP}}$ genes. The analyses of resistance genes using the WGS data (ABRicate) [11] identified alleles $b l a_{\mathrm{VIM}-2}$ and $b l a_{\mathrm{IMP}-18}$. Taken together, 4 out of the 8 strains exhibited both $b l a_{\mathrm{VIM}-2}$ and $b l a_{\mathrm{IMP}-18}$ alleles. The remaining 4 strains harbored only the $b l a_{\mathrm{VIM}-2}$ gene.

\section{Discussion}

Carbapenems constitute one of the final lines of defense against resistant bacteria, particularly Gram-negative bacilli. CRPA is ranked as "critical priority" for research and development for new drugs and to implement antimicrobial stewardship initiatives by the World Health Organization [13]. Detecting the mechanisms of resistance to antimicrobials provide a helpful tool to prevent and control HAIs, especially considering the growing evidence of mobile genetic elements mediating and exacerbating nosocomial outbreaks $[14,15]$. To address these critical pathogens, we implemented commercial rapid molecular tests, hybridization assays, and whole-genome sequencing to analyze CRPA in HAI and compare the aforementioned methods.

Eight of the $32 P$. aeruginosa isolates from HAI in the National Children's Hospital of Costa Rica exhibited carbapenem resistance. The presence of the $b l a_{\mathrm{VIM}}$ gene was identified using two different commercial kits. However, when testing the same isolates by PCR LDT, we also detected the $b l a_{\mathrm{IMP}}$ gene in addition to the $b l a_{\mathrm{VIM}}$ gene. WGS analyses confirmed the presence of alleles $b l a_{\mathrm{VIM}-2}$ and $b l a_{\text {IMP-18, }}$, which were previously described in one of the main hospitals treating adults in the country [16]. Both genes are known to encode metallo- $\beta$-lactamase that confer resistance to carbapenems. Our analyses provide valuable information about the circulation of $P$. aeruginosa carrying $b l a_{\mathrm{IMP}-18}$ and $b l a_{\mathrm{VIM}-2}$ alleles in pediatric infections in Costa Rica. Moreover, we identified that allele $b l a_{\mathrm{IMP}-18}$ is present in HAI, but not targeted in the current rapid molecular technologies available in Costa Rica. This could lead to miss-identification of the $P$. aeruginosa resistance mechanisms if healthcare facilities don't have access to redundant methods for confirmation.

This work brings together the context of hospitalacquired infections, antimicrobial stewardship, diagnostic stewardship, availability of diagnostic methods, and whole genome sequencing to highlight the importance of local epidemiology when adopting strategies to 
fight antimicrobial resistance. Similar situations can be observed globally as infections due to multidrug-resistant bacteria increase and new technologies become available. Rapid molecular tests for the detection of antibacterial resistance continue to expand around the world. The availability, presentation, multiplex format, international validation, and low price makes these kits very attractive to use in economically constrained countries. However, they are developed for high-income countries in accordance with their needs and epidemiological contexts; thus, they might not have all of the targets that are circulating in other countries.

\section{Conclusions}

The results of this study highlight the importance of knowing the local epidemiology when monitoring for CRPA in the context of HAIs using rapid molecular tests that were originally created for a different country.

\section{Abbreviations}

GLASS: The global antimicrobial resistance surveillance system; WGS: Wholegenome sequencing; CRPA: Carbapenem-resistant Pseudomonas aeruginosa; HAl: Hospital-acquired infections; CLSI: Clinical Laboratory Standards Institute; mCIM: Modified carbapenem inhibition test; LDT: Laboratory-developed tests; MBL: Metallo-ß-lactamase; PCR: Polimerase chain reaction.

\section{Acknowledgements}

Our special thanks to Drs. Tarah Lynch, Erin Symonds and Helena Brenes for their accurate suggestions on the preparation of this manuscript.

\section{Authors' contributions}

Dr. P-C contributed with the conception, development, molecular and sequencing assays and analyses of data and writing of the manuscript. Dr. P-B contributed with development, molecular and sequencing assays and draft review. Dr. M-A contributed with the phenotypic assays, culture and identification of strains and draft review. All authors read and approved the final manuscript.

\section{Funding}

The authors did not receive any funding for this work.

\section{Availability of data and materials}

The datasets used and/or analysed during the current study are available from the corresponding author on reasonable request.

\section{Declarations}

\section{Ethics approval and consent to participate}

This study has been authorized by the Investigation Review Board of the National Children's Hospital of Costa Rica, No. CEC-HNN-313-2020.

\section{Consent for publication}

Not applicable.

\section{Competing interests}

The authors declare that they have no competing interests with this publication.
Received: 6 January 2021 Accepted: 21 April 2021

Published online: 28 April 2021

\section{References}

1. World Health Organization. The global antimicrobial resistance surveillance system: microbiological laboratory standards and tools. 2020. www. who.int/glass/laboratory/en. Accessed 7 Dec 2020.

2. Global antimicrobial resistance surveillance system (GLASS) report: early implementation 2020. Geneva: World Health Organization. 2020. License CC-BY-NC-SA. 3.0 IGO.

3. Wenjing $W u$, Feng $Y u$, Tang G, et al. NDM metallo- $\beta$-lactamases and their bacterial producers in health care settings. Clin Microb Rev. 2019:32(2):e00115-e118.

4. Miller JM, Binnicker MJ, Campbell S, et al. A guide to utilization of the microbiology laboratory for diagnosis of infectious diseases: 2018 update by the infectious diseases society of America and the American society for microbiology. Clin Infect Dis. 2018;67(6):e1-94.

5. Clinical Laboratory Standards Institute (CLSI). Performance standards for antimicrobial susceptibility testing. CLSI supplement M100. 30th Ed. Pennsylvania, USA. 2020.

6. Senda K, Arakawa Y, Ichiyama S, et al. PCR detection of metallo-beta-lactamase gene (bla|MP) in gram-negative rods resistant to broad-spectrum beta-lactams. J Clin Microbiol. 1996;34(12):2909-13.

7. Andrews S. FastQC: a quality control tool for high throughput sequence data. 2010. http://www.bioinformatics.babraham.ac.uk/projects/fastqc. Accessed Jan 2021.

8. Bolger AM, Lohse M, Usadel B. Trimmomatic: a flexible trimmer for Illumina sequence data. Bioinformatics. 2014;1:1. https://doi.org/10.1093/ bioinformatics/btu170.

9. Seemann T. Shovill: faster SPAdes assembly of Illumina reads. (v1.0.4) 2017. https://github.com/tseemann/shovill. Accessed Jan 2021.

10. Seemann T. Prokka: rapid prokaryotic genome annotation. Bioinformatics 2014;30(14):2068-9. https://doi.org/10.1093/bioinformatics/btu153.

11. Seemann T. ABRicate: mass screening of contigs for antiobiotic resistance genes. v1.0.1. 2016. https://github.com/tseemann/abricate. Accessed Jan 2021.

12. Afgan E, Baker D, Batut B, et al. The Galaxy platform for accessible, reproducible and collaborative biomedical analyses: 2018 update. Nucleic Acids Res. 2018;46(W1):W537-44.

13. World Health Organization. Prioritization of pathogens to guide discovery, research and development of new antibiotics for drug-resistant bacterial infections including tuberculosis. 2017. pp. 12, 71.

14. van der Zwaluw K, de Haan A, Pluister GN, et al. The Carbapenem inactivation method (CIM), a simple and low-cost alternative for the Carba Np test to assess phenotypic Carbapenemase activity in gram-negative rods. PLoS ONE. 2015;10(3):e0123690.

15. Martin J, Phan HT, Findlay J, et al. Covert dissemination of carbapenemase-producing Klebsiella pneumoniae (KPC) in a successfully controlled outbreak: long- and short-read whole-genome sequencing demonstrate multiple genetic modes of transmission. J Clin Microbiol. 2015;72(11):3025-34.

16. Toval F, Guzmán-Marte A, Madriz V, et al. Predominance of carbapenemresistant Pseudomonas aeruginosa isolates carrying bla $a_{\mathrm{IMP}}$ and bla $a_{\mathrm{VIM}}$ metallo-beta-lactamases in a major hospital in Costa Rica. J Med Microbiol. 2015;64:37-43.

\section{Publisher's Note}

Springer Nature remains neutral with regard to jurisdictional claims in published maps and institutional affiliations. 